\title{
Genomic differentiation of Hordeum chilense from $H$. vulgare as revealed by repetitive and EST sequences
}

\author{
Adel Abdel-Aziz Hagras ${ }^{1}$, Masahiro Kishii ${ }^{1 \dagger}$, Hiroyuki Tanaka ${ }^{1}$, \\ Kazuhiro Sato ${ }^{2}$ and Hisashi Tsujimoto ${ }^{1 *}$ \\ ${ }^{1}$ Laboratory of Plant Genetics and Breeding Science, Faculty of Agriculture, Tottori \\ University, Tottori 680-8553, Japan \\ ${ }^{2}$ Research Institute for Bioresources, Okayama University, \\ Kurashiki 710-0046, Japan
}

(Received 31 March 2005, accepted 6 June 2005)

\begin{abstract}
Hordeum vulgare, cultivated barley, and its wild relative, $H$. chilense, have several important traits that might be useful for wheat improvement. Here, in situ hybridization and barley expressed sequence tag (EST) markers were used to characterize and compare the chromosomes of $H$. chilense with those of $H$. vulgare. FISH with four repetitive DNA sequences, AG, AAG, 5S rDNA and 45S rDNA, was applied to the mitotic chromosomes of $H$. vulgare, $H$. chilense and available wheat- $H$. chilense addition and substitution lines. FISH with the AAG repeat differentiated the individual chromosomes of $H$. chilense and $H$. vulgare. The patterns of FISH signals in the two species differed greatly. The 45S rDNA signals were observed on two pairs of chromosomes in both species, while the 5S rDNA signals were observed on four pairs of chromosomes in $H$. vulgare and on one pair in $H$. chilense. The AG repeat showed FISH signals at the centromeric regions of all chromosomes of $H$. vulgare but none of the chromosomes of $H$. chilense. These results indicate that the chromosomes of the two species are highly differentiated. To study the homoeology between the two species, 209 EST markers of $H$. vulgare were allocated to individual chromosomes of $H$. chilense. One hundred and forty of the EST markers were allocated to respective chromosomes of $H$. chilense using the wheat- $H$. chilense addition and substitution lines. Twenty-six EST markers on average were allocated to each chromosome except to the chromosome $2 \mathrm{H}^{\text {ch }} \mathrm{S}$, to which only 10 markers were allocated. Ninety percent of the allocated EST markers in $H$. chilense were placed on $H$. vulgare chromosomes of the same homoeologous group, indicating that the expressed sequences of the two species were highly conserved. These EST markers would be useful for detecting chromatin introgressed from these species into the wheat genome.
\end{abstract}

Key words: $H$. chilense, $H$. vulgare, alien chromosome addition lines, FISH, EST, homoeology

\section{INTRODUCTION}

Related, wild and progenitor species represent a rich reservoir of useful genetic variation that can be exploited for crop improvement. Interspecific and intergeneric hybridization in the tribe Triticeae have been successfully used to facilitate the transfer of genes from wild relatives to cultivated species. Many amphiploids between wheat

Edited by Takashi Endo

* Corresponding author. E-mail: tsujim@muses.tottori-u.ac.jp

${ }^{\dagger}$ Present address: Genetic Resources, CIMMYT, Apdo. Postal 6641, 06600 Mexico, D.F., Mexico and other species, and alien chromosome addition, substitution or translocation lines have been produced and maintained for this purpose. Agronomically important traits, including resistance to diseases, pests and abiotic stresses, have been transferred from these genetic stocks into wheat (Knott and Dvorak, 1976; Sharma and Gill, 1983; Gale and Miller, 1987; Jiang et al., 1994; Friebe et al., 1996).

Cytological and molecular characterization of alien chromosomes in a wheat genetic background would accelerate production of such genetic stocks and introgression of alien chromosome segments including useful genes into 
wheat. Fluorescence in situ hybridization (FISH) is well established as a highly efficient method for cytological characterization. It has been used for physical mapping of specific sequences in wheat and its relatives (Mukai et al., 1990 and 1992; Leitch et al., 1991; Jiang and Gill, 1994) to locate the breakpoints of chromosome translocations (Friebe et al., 1991; Mukai et al., 1993; Jiang et al., 1994, Molnar-Lang et al., 2000) and in karyotyping (Cabrera et al., 1995; Tsujimoto et al., 1997).

$H$. chilense is a wild species of Hordeum . However, its genome $\left(\mathrm{H}^{\mathrm{ch}}\right)$ and geographical distribution (South America) are quite different from those of cultivated barley, $H$. vulgare. The use of $H$. chilense in breeding programs has focused on the development of tritordeums (amphiploids between wheat and $H$. chilense) to be used as new cereals (Martín, 1998) and for the introgression of novel traits into wheat. The amphiploids could be used as a bridge for the genetic transfer of useful genes from $H$. chilense to wheat or barley (Martín et al., 1998). Many $H$. chilense novel traits have been allocated to specific chromosomes: resistance to root-rot nematode on chromosome $1 \mathrm{H}^{\text {ch }} \mathrm{S}$, tolerance to salt on chromosomes $1 \mathrm{H}^{\text {ch }}, 4 \mathrm{H}^{\text {ch }}$ and $5 \mathrm{H}^{\mathrm{ch}}$, resistance to Septoria on chromosome $4 \mathrm{H}^{\mathrm{ch}}$, and high carotenoid pigment content and resistance to common bunt both on chromosome $7 \mathrm{H}^{\mathrm{ch}}$ (Person-Dedryver et al., 1990, Martín et al., 2000). H. chilense chromosome addition lines of hexaploid wheat have been obtained (Miller et al., 1982) and characterized by C-banding and in situ hybridization (Cabrera et al., 1995). The addition lines could be very useful tools for the introgression of $H$. chilense chromatin in wheat if such introgressions could be easily and reliably characterized using genetic markers.

Recently, a total of 120,000 clones of barley expressed sequence tags (ESTs) have been sequenced and analyzed, and 10,336 primer sets developed by Sato et al. (2003). Of these primers, 5,133 sets were tested for polymorphism between barley (cv. Betzes) and bread wheat (cv. Chinese Spring). One-third of the tested primers showed a presence/absence type of polymorphism bet- ween wheat and barley. Previously, we tested this group of primers for polymorphisms between bread wheat and ten alien Triticeae species, including $H$. chilense (Hagras et al., 2005). A large number of such barley EST markers were effective in $H$. chilense and many of them were polymorphic between wheat and $H$. chilense. These markers can be used to identify DNA of $H$. chilense in a wheat genetic background. To examine the similarity of the chromosomes of $H$. chilense and $H$. vulgare, in this study we allocated most of these barley EST markers to specific chromosomes of $H$. chilense and compared the chromosome morphology between $H$. chilense and $H$. vulgare by FISH with four repetitive sequences.

\section{MATERIALS AND METHODS}

Plant materials Hordeum vulgare cv. Betzes $(2 n=2 x$ = 14; genome $\mathrm{HH}), H$. chilense $(2 n=2 x=14$; genome $\left.\mathrm{H}^{\mathrm{ch}} \mathrm{H}^{\mathrm{ch}}\right)$, common wheat cv. Chinese Spring $(2 n=6 x=42$; genome AABBDD) and six $H$. chilense addition and substitution lines of Chinese Spring wheat were used in this study (Table 1). The chromosome addition line of $2 \mathrm{H}^{\mathrm{ch}}$ that was used was addition of the short arm of $2 \mathrm{H}^{\mathrm{ch}}$. The $H$. chilense chromosome addition and substitution lines, produced by Miller et al. (1982), were introduced from Wheat Genetics Resource Center, Kansas State University. The plant materials used in this study have been maintained in Tottori Alien Chromosome Bank of Wheat (TACBOW), managed by the National Bioresources Project-Wheat, Japan.

Fluorescence in situ hybridization (FISH) FISH was carried out as described by Kishii et al. (1999). Collected root tips were pretreated with ice water for $24 \mathrm{~h}$ and fixed in acetic acid: ethanol (1:3) for more than 3 days at room temperature. Mitotic slides were prepared by the acetocarmine squash method. Then the slides were denatured in $0.15 \mathrm{~N} \mathrm{NaOH}$ (in $70 \%$ ethanol) for $5 \mathrm{~min}$ at room temperature, and dehydrated sequentially in cold $70 \%$, $90 \%$, and $99.5 \%$ ethanol. Five probes: $H$. chilense

Table 1. Plant materials and genomic constitutions used in this study

\begin{tabular}{lll}
\hline \hline Line & Genomic constitution & Accession number \\
\hline Triticum aestivum cv. 'Chinese Spring' & AABBDD & HT23S1 \\
Hordeum vulgare cv. 'Betzes' & $\mathrm{HH}$ & TACBOW0116 \\
H. chilense & $\mathrm{H}^{\mathrm{ch}} \mathrm{H}^{\mathrm{ch}}$ & TACBOW0115 \\
$1 \mathrm{H}^{\mathrm{ch}}$ disomic substitution line & AABBDD $-1^{\prime \prime \mathrm{a}}+1 \mathrm{H}^{\mathrm{ch} \prime}$ & TACBOW0051 \\
$2 \mathrm{H}^{\mathrm{ch}} \mathrm{S}$ ditelosomic addition line & AABBDD $+2 \mathrm{H}^{\mathrm{ch}} \mathrm{S}^{\prime \prime}$ & TACBOW0052 \\
$4 \mathrm{H}^{\mathrm{ch}}$ disomic substitution line & AABBDD $-1^{\prime \prime \mathrm{a}}+4 \mathrm{H}^{\mathrm{ch} \prime}$ & TACBOW0053 \\
$5 \mathrm{H}^{\mathrm{ch}}$ disomic addition line & AABBDD $+5 \mathrm{H}^{\mathrm{ch} \prime}$ & TACBOW0054 \\
$6 \mathrm{H}^{\mathrm{ch}}$ disomic addition line & AABBDD $+6 \mathrm{H}^{\mathrm{ch} \prime}$ & TACBOW0055 \\
$7 \mathrm{H}^{\mathrm{ch}}$ disomic addition line & AABBDD $+7 \mathrm{H}^{\mathrm{ch} \prime}$ & TACBOW0056 \\
\hline
\end{tabular}

a The substituted chromosomes of wheat are unknown. 
Table 2. Primer sequences and chromosomal locations of the EST markers on $H$. chilense chromosomes and on $H$. vulgare chromosomes

\begin{tabular}{|c|c|c|c|c|}
\hline \multirow{2}{*}{ EST marker } & \multirow{2}{*}{ Primer (L) } & \multirow{2}{*}{ Primer $(R)$} & \multicolumn{2}{|c|}{ Chromosomal location } \\
\hline & & & H. chilense & H. vulgare ${ }^{\mathrm{a}}$ \\
\hline BAWU12 & TCCAGACTCTCTGGCGCTAT & GGCGGGCTCTATGAACACTA & $1 \mathrm{H}^{\mathrm{ch}}$ & $1 \mathrm{H}(\mathrm{P}, \mathrm{G})$ \\
\hline BAWU136 & CAACCCAGCTACAGGGTGAT & CGTCATGTGCGTTCACCTAC & $1 \mathrm{H}^{\mathrm{ch}}$ & $1 \mathrm{H}(\mathrm{P}, \mathrm{G})$ \\
\hline BAWU162 & СCССATATCATCCTCССТTT & GCAGTAATGACCTTTCCCCA & $1 \mathrm{H}^{\mathrm{ch}}$ & $1 \mathrm{H}(\mathrm{P}, \mathrm{G})$ \\
\hline BAWU211 & AGCCTGTAGCAGCATGGATT & CACATCGACATGTTCCTTGG & $1 \mathrm{H}^{\mathrm{ch}}$ & $1 \mathrm{H}(\mathrm{P}, \mathrm{G})$ \\
\hline BAWU243 & ATCAAAGGAATGCAGGGTTG & TCAGTTTCATCGCACTCTGG & $1 \mathrm{H}^{\mathrm{ch}}$ & $1 \mathrm{H}(\mathrm{P}, \mathrm{G})$ \\
\hline BAWU316 & TAGCTGGTCACCTCGCTCTT & TGCTGCTTGACTCGTTCATC & $1 \mathrm{H}^{\mathrm{ch}}$ & $1 \mathrm{H}(\mathrm{P}, \mathrm{G})$ \\
\hline BAWU541 & CCGGTATTGACAAGCATCAA & ATTCATGGCACTCCGTCTTC & $1 \mathrm{H}^{\mathrm{ch}}$ & $1 \mathrm{H}(\mathrm{P}, \mathrm{G})$ \\
\hline BAWU585 & AAGGGGCCGAATTATTTTTG & ACGGACATAGGTCAAATCCG & $1 \mathrm{H}^{\mathrm{ch}}$ & $1 \mathrm{H}(\mathrm{P}, \mathrm{G})$ \\
\hline BAWU616 & CAAGCAAATCCTCACTGCAA & GACCTCAAGTCGTACCTGGC & $1 \mathrm{H}^{\mathrm{ch}}$ & $1 \mathrm{H}(\mathrm{P}, \mathrm{G})$ \\
\hline BAWU17 & GAACTGTGCCTTGCTCATCA & ACCTCGCTGCTAGAGCACAT & $1 \mathrm{H}^{\mathrm{ch}}$ & $1 \mathrm{H}(\mathrm{P})$ \\
\hline BAWU25 & TGTGTTTTATTCAGCTGGCG & TGCTCTTTCCTCACCAACCT & $1 \mathrm{H}^{\mathrm{ch}}$ & $1 \mathrm{H}(\mathrm{P})$ \\
\hline BAWU299 & ATCTGGTTTCCGTTCATTCG & TCACGCAACATTCCTCTCTG & $1 \mathrm{H}^{\mathrm{ch}}$ & $1 \mathrm{H}(\mathrm{P})$ \\
\hline BAWU366 & TGGGCTTAATTTCATCAGCC & GACCCTTTGAAGATCCGTGA & $1 \mathrm{H}^{\mathrm{ch}}$ & $1 \mathrm{H}(\mathrm{P})$ \\
\hline BAWU404 & GACGCTCTGAACGACAAACA & АCTTCATCAACTGCCTTCGC & $1 \mathrm{H}^{\mathrm{ch}}$ & $1 \mathrm{H}(\mathrm{P})$ \\
\hline BAWU498 & ACCCACTTTCGAAGCTCTCA & CCTGTGGCGAAAAAGAAGAG & $1 \mathrm{H}^{\mathrm{ch}}$ & $1 \mathrm{H}(\mathrm{P})$ \\
\hline BAWU574 & AAAACAGAACCGTGGAGTGG & AATGTGGTTGGCAGAAAAGG & $1 \mathrm{H}^{\text {ch }}$ & $1 \mathrm{H}(\mathrm{P})$ \\
\hline BAWU671 & CTGTTCGGACCAGTTGGTTT & ACAAAGAATGTCTGCGGGAC & $1 \mathrm{H}^{\mathrm{ch}}$ & $1 \mathrm{H}(\mathrm{P})$ \\
\hline BAWU735 & CTTGCTCAAAGTCGTTTCCA & CAGGCTTTACCGGAACATTG & $1 \mathrm{H}^{\mathrm{ch}}$ & $1 \mathrm{H}(\mathrm{P})$ \\
\hline BAWU756 & AGTTCCTCCTCTTGGTGGGT & ATCAGGACCCCTTCCTTCAT & $1 \mathrm{H}^{\mathrm{ch}}$ & $1 \mathrm{H}(\mathrm{P})$ \\
\hline BAWU760 & ACACGTTGGTGGCAGATACA & ACGAACATCTACGGAATGGC & $1 \mathrm{H}^{\mathrm{ch}}$ & $1 \mathrm{H}(\mathrm{P})$ \\
\hline BAWU532 & CCCAAACGTCCTTGTTCCTA & CGCAGGTAGCCAAAAATAGC & $1 \mathrm{H}^{\mathrm{ch}}$ & $2 \mathrm{H}(\mathrm{P}, \mathrm{G})$ \\
\hline BAWU785 & TCAGCTCCATCAACACTTCG & AGCTGGTGATAGGGCATTTG & $1 \mathrm{H}^{\mathrm{ch}}$ & $7 \mathrm{H}(\mathrm{P}, \mathrm{G})$ \\
\hline BAWU524 & GTTGCACGCAAACGTTTCTA & AGCTTGGCTATCTTGCTGGA & $1 \mathrm{H}^{\mathrm{ch}}$ & $4 \mathrm{H}, 5 \mathrm{H}, 6 \mathrm{H}(\mathrm{P})$ \\
\hline BAWU216 & CGGAACCACACACACAAACT & CCTTCCCTGGTGATTTGAAAC & $1 \mathrm{H}^{\mathrm{ch}}$ & - \\
\hline BAWU235 & TCAAGGCACAAGGCAGTATG & TCAATGCAAGTGAGGAACCA & $1 \mathrm{H}^{\mathrm{ch}}$ & - \\
\hline BAWU343 & TCGTACCATACAATGCTAACCG & TGCAGAGGTTTGAAGAAGCC & $1 \mathrm{H}^{\mathrm{ch}}$ & - \\
\hline BAWU719 & GACAGCCTTGCAGCAATACA & GTCAGCCATCTCACCCTCAT & $1 \mathrm{H}^{\mathrm{ch}}$ & _ \\
\hline BAWU842 & ATGTAGAACCCAATCGCGTC & CAAAATGAGCAAGCCTGTGA & $1 \mathrm{H}^{\mathrm{ch}}$ & - \\
\hline BAWU917 & GCGCCAGCTTTGTGTATGTA & ATGCTTCTGGGTAACGTTGG & $1 \mathrm{H}^{\mathrm{ch}}$ & - \\
\hline BAWU288 & TAGGGTTCGTCGCTGCTAGT & ACCAAAGAAGGAGGTGGCTT & $2 \mathrm{H}^{\mathrm{ch}}$ & $2 \mathrm{H}(\mathrm{P}, \mathrm{G})$ \\
\hline BAWU32 & GTTCGATCAGACATTCCGGT & TTTTCTCTAAACCCCCTCGC & $2 \mathrm{H}^{\mathrm{ch}}$ & $2 \mathrm{H}(\mathrm{P}, \mathrm{G})$ \\
\hline BAWU40 & CGGAGCTTCTGGTTGATTGT & AACTGCCAGTCCTTCCAATG & $2 \mathrm{H}^{\mathrm{ch}}$ & $2 \mathrm{H}(\mathrm{P}, \mathrm{G})$ \\
\hline BAWU759 & TCGACATCTCTCCCATTTCC & AACCAGATATGGATGCCAGG & $2 \mathrm{H}^{\mathrm{ch}}$ & $2 \mathrm{H}(\mathrm{P}, \mathrm{G})$ \\
\hline BAWU153 & ATGGGCCAATATCAATTCCA & TGCTGCGTTCAGCTTTAAGA & $2 \mathrm{H}^{\mathrm{ch}}$ & $2 \mathrm{H}(\mathrm{P})$ \\
\hline BAWU181 & TTTCGTGCCTTTGCTTCTTT & ATGGTTGCTGAAGTCCGAAG & $2 \mathrm{H}^{\mathrm{ch}}$ & $2 \mathrm{H}(\mathrm{P})$ \\
\hline BAWU555 & AAGAAATTGCGAGACGGAAG & TCCGTGTGAGTTTAGCATCG & $2 \mathrm{H}^{\mathrm{ch}}$ & $2 \mathrm{H}(\mathrm{P})$ \\
\hline BAWU660 & TGCAGCACACACTTCTTTCC & GATCGCTTCGGAGCATAGAC & $2 \mathrm{H}^{\mathrm{ch}}$ & $2 \mathrm{H}(\mathrm{P})$ \\
\hline BAWU387 & AGTTTTGTTGCCAAACGTCC & GCTTCATACCTTCGCCTCTG & $2 \mathrm{H}^{\mathrm{ch}}$ & $4 \mathrm{H}(\mathrm{P}, \mathrm{G})$ \\
\hline BAWU835 & GAAAAAGAAGCACGAGTCGG & CTCGCTGGAAACAGATCCAT & $2 \mathrm{H}^{\text {ch }}$ & - \\
\hline BAWU112 & GCACAACTGTCCCATTGTTG & GCCAAAGGAGGGAAAAACTC & $4 \mathrm{H}^{\mathrm{ch}}$ & $4 \mathrm{H}(\mathrm{P}, \mathrm{G})$ \\
\hline BAWU303 & AATGTGCCTCCACAGGGTAG & GATACTGAGTGGAAAGCGGC & $4 \mathrm{H}^{\mathrm{ch}}$ & $4 \mathrm{H}(\mathrm{P}, \mathrm{G})$ \\
\hline BAWU618 & AGATCGACACAAGGCCATTC & GAGCTTGGAGCTAATGACCG & $4 \mathrm{H}^{\mathrm{ch}}$ & $4 \mathrm{H}(\mathrm{P}, \mathrm{G})$ \\
\hline BAWU670 & CAACCTAGCATCGCTCATCA & ATTCGTCAAGAAATGGGCAC & $4 \mathrm{H}^{\mathrm{ch}}$ & $4 \mathrm{H}(\mathrm{P}, \mathrm{G})$ \\
\hline BAWU755 & TTCCGGACTATGAAACCCTG & TTGTTCGCTGCTTACAGTGG & $4 \mathrm{H}^{\mathrm{ch}}$ & $4 \mathrm{H}(\mathrm{P}, \mathrm{G})$ \\
\hline BAWU766 & AAACGGGAAATGTTTTGCAG & TCAAGAAGGCCATCGAGAAG & $4 \mathrm{H}^{\mathrm{ch}}$ & $4 \mathrm{H}(\mathrm{P}, \mathrm{G})$ \\
\hline BAWU915 & AGGCGTCCTATGGACACAAC & CCCTCGACACGACCATCTAT & $4 \mathrm{H}^{\mathrm{ch}}$ & $4 \mathrm{H}(\mathrm{P}, \mathrm{G})$ \\
\hline BAWU118 & CCAGTTCTTCTTGAGCAGGG & GTCTGGATTCCATGGACGTT & $4 \mathrm{H}^{\mathrm{ch}}$ & $4 \mathrm{H}(\mathrm{P})$ \\
\hline BAWU152 & GATGGTTGTGCTGCTGCTTA & AGCTCCTAAACATGGACCGA & $4 \mathrm{H}^{\mathrm{ch}}$ & $4 \mathrm{H}(\mathrm{P})$ \\
\hline BAWU171 & ATAACACGGGCAGAATTTCG & GGTTACTGCCGTGTTTGGTT & $4 \mathrm{H}^{\mathrm{ch}}$ & $4 \mathrm{H}(\mathrm{P})$ \\
\hline
\end{tabular}


Continued

\begin{tabular}{|c|c|c|c|c|}
\hline \multirow{2}{*}{ EST marker } & \multirow{2}{*}{ Primer $(\mathrm{L})$} & \multirow{2}{*}{ Primer $(\mathrm{R})$} & \multicolumn{2}{|c|}{ Chromosomal location } \\
\hline & & & H. chilense & H. vulgare ${ }^{\mathrm{a}}$ \\
\hline BAWU217 & CGCCCAATAAACCATTAAGC & CATCATCCAGGTGGTCTTCC & $4 \mathrm{H}^{\mathrm{ch}}$ & $4 \mathrm{H}(\mathrm{P})$ \\
\hline BAWU265 & TACGACGCGCATACTTCATC & ACATCATAGACCCTGCCCTG & $4 \mathrm{H}^{\mathrm{ch}}$ & $4 \mathrm{H}(\mathrm{P})$ \\
\hline BAWU312 & ACATTGGAGGATTCGCAAAG & ATCTCAGGCAACCAAACTGC & $4 \mathrm{H}^{\mathrm{ch}}$ & $4 \mathrm{H}(\mathrm{P})$ \\
\hline BAWU473 & CACCTCATCCATCCTTGCTT & TTGTCATGAACGCATGATCC & $4 \mathrm{H}^{\mathrm{ch}}$ & $4 \mathrm{H}(\mathrm{P})$ \\
\hline BAWU497 & TGTGCATCCCTCAAAACAAA & TTGCTCACAAAGTGCTCCTG & $4 \mathrm{H}^{\mathrm{ch}}$ & $4 \mathrm{H}(\mathrm{P})$ \\
\hline BAWU505 & ATGGAGGGGATAAATACGGC & TCTGTAATGCTTGTGGCCTG & $4 \mathrm{H}^{\mathrm{ch}}$ & $4 \mathrm{H}(\mathrm{P})$ \\
\hline BAWU507 & CACACCTTTTCCGTCCAAGT & TTATGGTAGCATGGCGAGTG & $4 \mathrm{H}^{\mathrm{ch}}$ & $4 \mathrm{H}(\mathrm{P})$ \\
\hline BAWU552 & CCCCTGACAGCCTACAGCTA & AGGAGATGGTGTCCAGATCG & $4 \mathrm{H}^{\mathrm{ch}}$ & $4 \mathrm{H}(\mathrm{P})$ \\
\hline BAWU673 & AATGGGATCCATTCCCGTAT & AACGTCCTCCGTTTCATCAC & $4 \mathrm{H}^{\mathrm{ch}}$ & $4 \mathrm{H}(\mathrm{P})$ \\
\hline BAWU686 & GAAGAGACCGTGCCAAGTGT & CACGGAGGCAACAATCTGTA & $4 \mathrm{H}^{\mathrm{ch}}$ & $4 \mathrm{H}(\mathrm{P})$ \\
\hline BAWU720 & GCAACCCCTAACATTGCCTA & TCCTGGCATGCAGTCAATTA & $4 \mathrm{H}^{\mathrm{ch}}$ & $4 \mathrm{H}(\mathrm{P})$ \\
\hline BAWU808 & TGCCCCCAAACTTTATATGC & GAGGGTCTTCCTGTTGTGGA & $4 \mathrm{H}^{\mathrm{ch}}$ & $4 \mathrm{H}(\mathrm{P})$ \\
\hline BAWU852 & ATCTCCACGCTAGCAGAACC & GCTCTGATGGCAATGATCTG & $4 \mathrm{H}^{\mathrm{ch}}$ & - \\
\hline BAWU884 & GGACACGAGCTTCGTTTTTC & TAGCTGCATTGCTCAACTGG & $4 \mathrm{H}^{\mathrm{ch}}$ & - \\
\hline BAWU886 & CGTACCTCGACAAAAGCACA & GTGTAAATGGCCCAGATGCT & $4 \mathrm{H}^{\mathrm{ch}}$ & - \\
\hline BAWU898 & ACATATTCTCCCGTGGTTGG & GCATTGCCAACGGTAAAGAT & $4 \mathrm{H}^{\mathrm{ch}}$ & - \\
\hline BAWU15 & TTGCAGGTACTCCGGGTAAT & TTGTGCGATCGGTTCCTACT & $5 \mathrm{H}^{\mathrm{ch}}$ & $5 \mathrm{H}(\mathrm{P}, \mathrm{G})$ \\
\hline BAWU160 & GCAACCGTAGTCCTTGTGGT & GTGTGGCCTTTCAGGTGATT & $5 \mathrm{H}^{\mathrm{ch}}$ & $5 \mathrm{H}(\mathrm{P}, \mathrm{G})$ \\
\hline BAWU227 & CCAGACTGACGATTTGGGAT & GTGAAGCAATTCATGCAAGC & $5 \mathrm{H}^{\mathrm{ch}}$ & $5 \mathrm{H}(\mathrm{P}, \mathrm{G})$ \\
\hline BAWU240 & AGGTCATGCAGCTCTGTTCC & TGCGGGAGACCTACTTCAAC & $5 \mathrm{H}^{\mathrm{ch}}$ & $5 \mathrm{H}(\mathrm{P}, \mathrm{G})$ \\
\hline BAWU271 & TGCACAAGAACAGTGCATCA & TTGAGGAGCTTCTGGCATTT & $5 \mathrm{H}^{\mathrm{ch}}$ & $5 \mathrm{H}(\mathrm{P}, \mathrm{G})$ \\
\hline BAWU315 & GTACCATTAGCTGCCCCAAA & GGCATGCTCTTCGAGTTTTC & $5 \mathrm{H}^{\mathrm{ch}}$ & $5 \mathrm{H}(\mathrm{P}, \mathrm{G})$ \\
\hline BAWU321 & CAGATGGCAGGCAATACAGA & ATAGCTTAACTGCCGCCGTA & $5 \mathrm{H}^{\mathrm{ch}}$ & $5 \mathrm{H}(\mathrm{P}, \mathrm{G})$ \\
\hline BAWU749 & CTTCATCGCAACTTGTGTGG & GAAGTACACGACCGCATTGA & $5 \mathrm{H}^{\mathrm{ch}}$ & $5 \mathrm{H}(\mathrm{P}, \mathrm{G})$ \\
\hline BAWU834 & AACCACAGGCACACATTCAA & AGGATACAACCCAAGCATCG & $5 \mathrm{H}^{\mathrm{ch}}$ & $5 \mathrm{H}(\mathrm{P}, \mathrm{G})$ \\
\hline BAWU891 & ACTTGCACACCCCAGCTAAT & TGCACAGCTACTGATTTGGC & $5 \mathrm{H}^{\mathrm{ch}}$ & $5 \mathrm{H}(\mathrm{P}, \mathrm{G})$ \\
\hline BAWU116 & CGATGAGTGTATACGTGCGG & GAACACATCTTCACCGAGGG & $5 \mathrm{H}^{\mathrm{ch}}$ & $5 \mathrm{H}(\mathrm{P})$ \\
\hline BAWU131 & GAACGCCAGCCAAATTGTAT & ACCATTTTGATCCTTCTGCG & $5 \mathrm{H}^{\mathrm{ch}}$ & $5 \mathrm{H}(\mathrm{P})$ \\
\hline BAWU30 & AACTGAAAAGTTGCCATCCG & GACCACAAAGCCGATGTTTT & $5 \mathrm{H}^{\mathrm{ch}}$ & $5 \mathrm{H}(\mathrm{P})$ \\
\hline BAWU362 & TACATTAGGACCGCTGGGAC & AGGGCAAGTCATTTACCACG & $5 \mathrm{H}^{\mathrm{ch}}$ & $5 \mathrm{H}(\mathrm{P})$ \\
\hline BAWU372 & GTGCATAGGGCAGATTCCAT & CCAGCTCAAGTTCCAGAGGA & $5 \mathrm{H}^{\mathrm{ch}}$ & $5 \mathrm{H}(\mathrm{P})$ \\
\hline BAWU423 & AGGGTGCTGCCATGTTTTAC & CGCCTGTGAGACAAGAATGA & $5 \mathrm{H}^{\mathrm{ch}}$ & $5 \mathrm{H}(\mathrm{P})$ \\
\hline BAWU494 & CCGGGGATGAAATAACAGAA & GCAGCATACAAGGCTGTTGA & $5 \mathrm{H}^{\mathrm{ch}}$ & $5 \mathrm{H}(\mathrm{P})$ \\
\hline BAWU495 & CGACGCCTAAGGTACATTTCA & CAGATGAAGGGAAGGCTCAA & $5 \mathrm{H}^{\mathrm{ch}}$ & $5 \mathrm{H}(\mathrm{P})$ \\
\hline BAWU651 & TTAAACAGCAAAAACGTGCG & AGTGGAAAGGATTTTGTGCG & $5 \mathrm{H}^{\mathrm{ch}}$ & $5 \mathrm{H}(\mathrm{P})$ \\
\hline BAWU780 & ACTCGCATGATACATCCACG & TAGATGGGGTCTCTCATGGC & $5 \mathrm{H}^{\mathrm{ch}}$ & $5 \mathrm{H}(\mathrm{P})$ \\
\hline BAWU782 & CAACTTGGACAACACAACGC & CTTGTGCATGCGCAGAGTAT & $5 \mathrm{H}^{\mathrm{ch}}$ & $5 \mathrm{H}(\mathrm{P})$ \\
\hline BAWU827 & GCCAAGCTCATATAGGGCAG & TATCTGTGATGCCACATCCG & $5 \mathrm{H}^{\mathrm{ch}}$ & $2 \mathrm{H}(\mathrm{P})$ \\
\hline BAWU20 & ACCCCGGAAGCTAAGATGAT & AGTCGGAACATGCGGTACAC & $5 \mathrm{H}^{\mathrm{ch}}$ & $3 \mathrm{H}(\mathrm{P}, \mathrm{G})$ \\
\hline BAWU713 & TGATCGTCGTAGAAAGCCCT & AGGTGCCACATTTTCCAGAC & $5 \mathrm{H}^{\mathrm{ch}}$ & - \\
\hline BAWU873 & ATCACAATTGGGTTGCCTTC & CACCAATCGTTTGGAGAGGT & $5 \mathrm{H}^{\mathrm{ch}}$ & - \\
\hline BAWU878 & GGGAACATACCAGAGTCGGA & AGAGCTGTTCTCAAGCTGCC & $5 \mathrm{H}^{\mathrm{ch}}$ & - \\
\hline BAWU155 & TCGACCACATGATCCACAGT & AGAACAACCACTACGACGGC & $6 \mathrm{H}^{\mathrm{ch}}$ & $6 \mathrm{H}(\mathrm{P}, \mathrm{G})$ \\
\hline BAWU29 & CTCGGGCAACAAAGTAGCTC & AGAAGGTGATGGTACCGCTG & $6 \mathrm{H}^{\mathrm{ch}}$ & $6 \mathrm{H}(\mathrm{P}, \mathrm{G})$ \\
\hline BAWU409 & ACCTCAAGCAAACTGCAACC & GAAAGTGCGTGAGGAGAAGC & $6 \mathrm{H}^{\mathrm{ch}}$ & $6 \mathrm{H}(\mathrm{P}, \mathrm{G})$ \\
\hline BAWU894 & CCTGCAGCTCTTCATGGTTT & ATTTTGCCACTACGGCTGTC & $6 \mathrm{H}^{\mathrm{ch}}$ & $6 \mathrm{H}(\mathrm{P}, \mathrm{G})$ \\
\hline BAWU107 & CGCCTATTTCTGAGCTCCTG & CGAGTATGGGAGTGGCAGTT & $6 \mathrm{H}^{\mathrm{ch}}$ & $6 \mathrm{H}(\mathrm{P})$ \\
\hline BAWU139 & TGATCTTTGGCAGAGCACAC & GGTTGGTGGGGAGTTGTATG & $6 \mathrm{H}^{\mathrm{ch}}$ & $6 \mathrm{H}(\mathrm{P})$ \\
\hline BAWU280 & TCTAAGCTAAAGCGACCCCA & CGAGAAAACTTCGGAGCATC & $6 \mathrm{H}^{\mathrm{ch}}$ & $6 \mathrm{H}(\mathrm{P})$ \\
\hline BAWU349 & AGCTAAGCTGCTTCCACTGC & TGAAGAAGTGAACGTGCTGG & $6 \mathrm{H}^{\mathrm{ch}}$ & $6 \mathrm{H}(\mathrm{P})$ \\
\hline
\end{tabular}


Continued

\begin{tabular}{|c|c|c|c|c|}
\hline \multirow{2}{*}{ EST marker } & \multirow{2}{*}{ Primer (L) } & \multirow{2}{*}{ Primer (R) } & \multicolumn{2}{|c|}{ Chromosomal location } \\
\hline & & & H. chilense & H. vulgare ${ }^{\mathrm{a}}$ \\
\hline BAWU361 & GCTGCATGCTGGACTCAATA & GCACTCATTGAACAGCAGGA & $6 \mathrm{H}^{\mathrm{ch}}$ & $6 \mathrm{H}(\mathrm{P})$ \\
\hline BAWU591 & AACAAGCAGCCAGATCACAG & CCATTCGGTCTTGACCAGTT & $6 \mathrm{H}^{\mathrm{ch}}$ & $6 \mathrm{H}(\mathrm{P})$ \\
\hline BAWU599 & GCAGCTGGGGGATATACAGA & CATCTCCATCATCATCGTCG & $6 \mathrm{H}^{\mathrm{ch}}$ & $6 \mathrm{H}(\mathrm{P})$ \\
\hline BAWU637 & AGAAACCAGGTCCAGCCTTT & GGTTACACATGGCGGAAACT & $6 \mathrm{H}^{\mathrm{ch}}$ & $6 \mathrm{H}(\mathrm{P})$ \\
\hline BAWU640 & GAAAATACATCGCGGGAAAA & TTAGCGTCCAGTCATCATGC & $6 \mathrm{H}^{\mathrm{ch}}$ & $6 \mathrm{H}(\mathrm{P})$ \\
\hline BAWU658 & TGTGGACGTGACAATCCCTA & AACAGAGGAAGAGGAGGGGA & $6 \mathrm{H}^{\mathrm{ch}}$ & $6 \mathrm{H}(\mathrm{P})$ \\
\hline BAWU94 & TTTCAAGCAGAGCTGCAAAG & GCTTGCTGAGCGCTTTCTAC & $6 \mathrm{H}^{\mathrm{ch}}$ & $6 \mathrm{H}(\mathrm{P})$ \\
\hline BAWU267 & ATCTGATGAACCCGAACCAC & ATCAACAAGCATCCAGGGAG & $6 \mathrm{H}^{\mathrm{ch}}$ & $3 \mathrm{H}(\mathrm{P}), 4 \mathrm{H}(\mathrm{G})$ \\
\hline BAWU233 & AAGCATAGCCTTGTCGGTTG & GGCAGATTTGCCATCTTCAT & $6 \mathrm{H}^{\mathrm{ch}}$ & $3 \mathrm{H}(\mathrm{P}, \mathrm{G})$ \\
\hline BAWU57 & GTGGACTGGGGATACCAATG & ATCAAGGATGGCTGGAGATG & $6 \mathrm{H}^{\mathrm{ch}}$ & $7 \mathrm{H}(\mathrm{P})$ \\
\hline BAWU346 & TCTTCCCTTCACTGGATGCT & TCAAGCGTTTCTGTGGTTTG & $6 \mathrm{H}^{\mathrm{ch}}$ & - \\
\hline BAWU892 & TCCCACGTTGGTCACTGTTA & TTGATATGTGCAAGCGGAAG & $6 \mathrm{H}^{\mathrm{ch}}$ & - \\
\hline BAWU104 & GGCACCAGGTGAGGAGATTA & TGCACTTTCTGCCTATGTGC & $7 \mathrm{H}^{\mathrm{ch}}$ & $7 \mathrm{H}(\mathrm{P}, \mathrm{G})$ \\
\hline BAWU123 & GCGGAGGACATACACCCTAA & AAGCTCAAGGGCGAGTACAA & $7 \mathrm{H}^{\mathrm{ch}}$ & $7 \mathrm{H}(\mathrm{P}, \mathrm{G})$ \\
\hline BAWU21 & CATGCGACACCTACGAGAGA & GAGAGTCACAGATGGCCACA & $7 \mathrm{H}^{\mathrm{ch}}$ & $7 \mathrm{H}(\mathrm{P}, \mathrm{G})$ \\
\hline BAWU302 & CATCCAATCTCAAATCCGCT & GGCAAAGCAATGCAAAAATC & $7 \mathrm{H}^{\mathrm{ch}}$ & $7 \mathrm{H}(\mathrm{P}, \mathrm{G})$ \\
\hline BAWU522 & GCGACCACACCCGATATTTA & AAGGACGAGGCACGAAGTTA & $7 \mathrm{H}^{\mathrm{ch}}$ & $7 \mathrm{H}(\mathrm{P}, \mathrm{G})$ \\
\hline BAWU576 & TGTGGAATGAGTGGGAACAA & GGTTGGATCCGCTAGCTACA & $7 \mathrm{H}^{\mathrm{ch}}$ & $7 \mathrm{H}(\mathrm{P}, \mathrm{G})$ \\
\hline BAWU631 & TACAACAACAATGGGGAGCA & TGAACTTGTGTCAGGGACCA & $7 \mathrm{H}^{\mathrm{ch}}$ & $7 \mathrm{H}(\mathrm{P}, \mathrm{G})$ \\
\hline BAWU680 & CCCGTCACATGTATCACTGC & CGTGGACATTGAGAGGGAGT & $7 \mathrm{H}^{\mathrm{ch}}$ & $7 \mathrm{H}(\mathrm{P}, \mathrm{G})$ \\
\hline BAWU709 & CCATAGACCAGCATCCCCTA & CATGTCGAGCAAGAAGACGA & $7 \mathrm{H}^{\mathrm{ch}}$ & $7 \mathrm{H}(\mathrm{P}, \mathrm{G})$ \\
\hline BAWU165 & ATGAGCTCGGCAAAAATGAG & AGGGATTCCATGTCGTTCAC & $7 \mathrm{H}^{\mathrm{ch}}$ & $7 \mathrm{H}(\mathrm{P})$ \\
\hline BAWU166 & TCGCAGTTACAACATCTCGC & CATGACCGCAAAGCTTGATA & $7 \mathrm{H}^{\mathrm{ch}}$ & $7 \mathrm{H}(\mathrm{P})$ \\
\hline BAWU379 & TGCTGCACAAAGCATAGGAC & AATGTGAAGGCACATGACGA & $7 \mathrm{H}^{\mathrm{ch}}$ & $7 \mathrm{H}(\mathrm{P})$ \\
\hline BAWU406 & GGTTCAGGGACGAAGGTACA & CTACGCCTAGCGGAGATGAG & $7 \mathrm{H}^{\mathrm{ch}}$ & $7 \mathrm{H}(\mathrm{P})$ \\
\hline BAWU517 & TTGCGATGATGATGGAGTGT & CTTTTCGTCTTTCTTTGCGG & $7 \mathrm{H}^{\mathrm{ch}}$ & $7 \mathrm{H}(\mathrm{P})$ \\
\hline BAWU550 & ATGCCACCATTTACAAAGCC & TTTCTGGGTCCTGATCCTTG & $7 \mathrm{H}^{\mathrm{ch}}$ & $7 \mathrm{H}(\mathrm{P})$ \\
\hline BAWU566 & TCATATCAAACCTCCGGGTC & TGCCAAGTTTATCCGAAACC & $7 \mathrm{H}^{\mathrm{ch}}$ & $7 \mathrm{H}(\mathrm{P})$ \\
\hline BAWU763 & AGAACCGAGATGAGGAATGTG & AGTCTCTTCGCGGAATCAAG & $7 \mathrm{H}^{\mathrm{ch}}$ & $7 \mathrm{H}(\mathrm{P})$ \\
\hline BAWU764 & TGCGGGAAATGGAGAATAAG & GGCTACAAGGACACCCGATA & $7 \mathrm{H}^{\mathrm{ch}}$ & $7 \mathrm{H}(\mathrm{P})$ \\
\hline BAWU79 & TTGTACGCCTGTCTTGCATC & AACAAGGGTCAACCGATGAG & $7 \mathrm{H}^{\mathrm{ch}}$ & $7 \mathrm{H}(\mathrm{P})$ \\
\hline BAWU829 & GAAAAGGTTACATGGGACGC & GGGACAACAGCACCTTTCAT & $7 \mathrm{H}^{\mathrm{ch}}$ & $7 \mathrm{H}(\mathrm{P})$ \\
\hline BAWU381 & CACATTAGCAAGCGCACAAT & AATTGATCAACCCTTGCCTG & $7 \mathrm{H}^{\mathrm{ch}}$ & $4 \mathrm{H}(\mathrm{P}, \mathrm{G})$ \\
\hline BAWU752 & TAGTACATGCGTGCCATGCT & GTCATCATCCTCGCCTTCAC & $7 \mathrm{H}^{\mathrm{ch}}$ & $5 \mathrm{H}(\mathrm{P}, \mathrm{G})$ \\
\hline BAWU559 & CCCAAAATTAAGGGCCAGAT & GGGACAAACTTGCTTGGAAA & $7 \mathrm{H}^{\mathrm{ch}}$ & $5 \mathrm{H}(\mathrm{P})$ \\
\hline BAWU127 & CGAATAGTGCCCGAAAACAT & TCCATCCCCAGCTAGAAGAA & $7 \mathrm{H}^{\mathrm{ch}}$ & - \\
\hline BAWU514 & GCTTGAGGGTCTTGTTCTGC & CAAGCTGGAAAAGGAAGCTG & $7 \mathrm{H}^{\mathrm{ch}}$ & - \\
\hline BAWU681 & GTCACAATTTGCATTGCAGG & GCGTGGAGGAGATATCGTGT & $7 \mathrm{H}^{\mathrm{ch}}$ & - \\
\hline BAWU857 & AGCTCAAGCCTTTGCAGCTA & TGTTTCAGGTGGTGTTGCAT & $7 \mathrm{H}^{\mathrm{ch}}$ & - \\
\hline BAWU872 & AGTGTCTATTTTGGTGCCGC & CATCTTGCTGGACAGGGATT & $7 \mathrm{H}^{\mathrm{ch}}$ & - \\
\hline BAWU879 & ACGAAAACCTCGAGTGGAGA & AGCTTGTGGCTGCAAAGAAT & $7 \mathrm{H}^{\mathrm{ch}}$ & - \\
\hline BAWU880 & TCTTACATCACCAGCGAGGA & AGAAGTTCGCCTACACCGC & $1 \mathrm{H}^{\mathrm{ch}}, 4 \mathrm{H}^{\mathrm{ch}}$ & - \\
\hline BAWU108 & AGGGGCTCATGATACATTCG & GATCGCATGATGATGACAGG & $1 \mathrm{H}^{\mathrm{ch}}, 2 \mathrm{H}^{\mathrm{ch}}, 5 \mathrm{H}^{\mathrm{ch}}$ & $4 \mathrm{H}(\mathrm{P})$ \\
\hline BAWU114 & TGGACGTGGTGAGACTACGA & TACCGTATGGGCTCTTCCTG & $1 \mathrm{H}^{\mathrm{ch}} 6 \mathrm{H}^{\mathrm{ch}}$ & $2 \mathrm{H}(\mathrm{P}, \mathrm{G})$ \\
\hline BAWU167 & ATTTACAGGAACTGTGGCGG & GTTGCACCTGGTTCCCTAGA & $6 \mathrm{H}^{\mathrm{ch}}, 7 \mathrm{H}^{\mathrm{ch}}$ & $6 \mathrm{H}(\mathrm{P})$ \\
\hline BAWU310 & ATGGTCTGCATGGTGAAACA & TCAAACGCTGCAAAACTGAC & $2 \mathrm{H}^{\mathrm{ch}}, 6 \mathrm{H}^{\mathrm{ch}}, 7 \mathrm{H}^{\mathrm{ch}}$ & $3 \mathrm{H}, 6 \mathrm{H}(\mathrm{P})$ \\
\hline BAWU577 & TACATTGGTGGACTTCGCTG & AGCCAGGCTATTGCTTCAAA & $1 \mathrm{H}^{\mathrm{ch}}, 5 \mathrm{H}^{\mathrm{ch}}$ & $7 \mathrm{H}(\mathrm{P}, \mathrm{G})$ \\
\hline BAWU707 & GCTTCTTCTGCCCAAGTGAG & CATGAGCAATCCTCTGGGTT & $4 \mathrm{H}^{\mathrm{ch}}, 6 \mathrm{H}^{\mathrm{ch}}$ & $4 \mathrm{H}, 5 \mathrm{H}, 6 \mathrm{H}, 7 \mathrm{H}(\mathrm{P})$ \\
\hline
\end{tabular}

\footnotetext{
${ }^{\text {a }}$ In $H$. vulgare, chromosomal locations of the ESTs were determined physically (P) by using wheat- $H$. vulgare addition lines
} (Nasuda et al., 2005) and genetically (G) by linkage analysis (Sato et al., unpublished data). 
genomic DNA, and AG, AAG, 5S rDNA and 45S rDNA repeats were used. The microsatellite sequences composed of AG and AAG repeats are organized in long tracts of heterochromatic DNA and are utilized as chromosome markers in some species of Triticeae (Pedersen et al., 1996; Hudakova et al., 2001). Sonicated genomic DNA and $45 \mathrm{~S}$ rDNA probes were labeled by the random primer labeling method with rhodamine-5-dUTP or FITC -11dUTP. The AG and AAG repeats were labeled by the 3' end labeling method with rhodamine-5-dUTP or FITC 11-dUTP using oligonucleotide primers $(\mathrm{AG})_{15}$ and $(\mathrm{AAG})_{10}$ as templates, respectively. The $5 \mathrm{~S}$ rDNA was labeled by the PCR method with biotin-16-dUTP. A hybridization solution (50\% formamide, $10 \%$ dextran sulfate, $1 \mu \mathrm{g} / \mu \mathrm{l}$ sonicated salmon sperm DNA, 50-500 $\mathrm{ng}$ of labeled probe, $2 \times \mathrm{SSC}$ ) was applied to each slide. For AG, AAG and $45 \mathrm{~S}$ rDNA probes, after hybridization at $37^{\circ} \mathrm{C}$ for $12-16 \mathrm{~h}$, the slides were washed in $2 \times \mathrm{SSC}$ at room temperature for $5 \mathrm{~min}$, briefly dried, and mounted with Vectashield (Vector) containing $1 \mu \mathrm{g} / \mu \mathrm{l}$ 4,6-diamidino-2-phenylindole (DAPI) for counterstaining of chromosomes. For the 5S rDNA probe, after hybridization at $37^{\circ} \mathrm{C}$ for $12-16 \mathrm{~h}$, the slides were washed in $2 \times$ $\mathrm{SSC}$ at room temperature for $5 \mathrm{~min}, 50 \%$ formamide in 2 $\times \mathrm{SSC}$ at $37^{\circ} \mathrm{C}$ for $15 \mathrm{~min}$, and $4 \times \mathrm{SSC}$ at room temperature for $5 \mathrm{~min}$, and then $120 \mu \mathrm{l}$ of a solution containing $200 \mu \mathrm{g} / \mu \mathrm{l}$ anti-digoxigenin-rhodamine (Roche) diluted with $1 \%$ bovine serum albumin (BSA) in $4 \times$ SSC was applied. After incubation for $30 \mathrm{~min}$ at $37^{\circ} \mathrm{C}$, the slides were washed in $2 \times \mathrm{SSC}, 0.1 \%$ TritonX-100 (in $2 \times \mathrm{SSC}$ ) and $2 \times \mathrm{SSC}$ at room temperature for $10 \mathrm{~min}$ each, briefly dried, and mounted with Vectashield containing $1 \mu \mathrm{g} / \mu \mathrm{l}$ DAPI. Using a fluorescence microscope (Olympus), photographs were taken with a cooled CCD camera (Photometrics).

DNA isolation and PCR Total genomic DNA was extracted from young plant leaves using the CTAB method (Murray and Thompson, 1980). PCR was performed in a 96-well plate, each well containing a $10-\mu l$ reaction mixture of $30 \mathrm{ng}$ of template DNA, 5 pmol of forward and reverse EST primers, $1 \mu \mathrm{l}$ of $10 \times E x$ Taq buffer $\left(20 \mathrm{mM} \mathrm{Mg}^{2+}\right), 0.8 \mu \mathrm{l}$ of dNTP mixture $(2.5 \mathrm{mM}$ each), and $0.25 \mathrm{U}$ of TaKaRa Ex Taq DNA polymerase (TaKaRa, Japan). After an initial denaturation step at $94^{\circ} \mathrm{C}$ for 2 $\mathrm{min}$, reactions were subjected to a touch-down amplification: 5 cycles at $94^{\circ} \mathrm{C}$ for $30 \mathrm{sec}, 65^{\circ} \mathrm{C}$ for $30 \mathrm{sec}$ (decreasing $1^{\circ} \mathrm{C} /$ cycle), $72^{\circ} \mathrm{C}$ for $2 \mathrm{~min}$ and subsequent 35 cycles at $94^{\circ} \mathrm{C}$ for $30 \mathrm{sec}, 60^{\circ} \mathrm{C}$ for $30 \mathrm{sec}, 72^{\circ} \mathrm{C}$ for $2 \mathrm{~min}$, with a final extension step at $72^{\circ} \mathrm{C}$ for $7 \mathrm{~min}$. Electrophoresis of amplification products was performed in $1.5 \%$ agarose gels.

Barley EST primers Two hundred and nine barley EST primer sets were used. The primer names and base sequences are presented in Table 2. These primers were previously screened for polymorphisms (presence/absence or size polymorphisms) between wheat and $H$. chilense (Hagras et al., 2005). Therefore, they would be expected to amplify markers of $H$. chilense in a wheat genetic background. Moreover, most of these markers have already been allocated to $H$. vulgare chromosomes using wheat- $H$. vulgare addition lines (Nasuda et al., 2005), a linkage map (Sato et al., unpublished data), or both.

\section{RESULTS}

Fig. 1 shows root-tip metaphase spreads of $H$. chilense and $H$. vulgare hybridized with AAG, 5S rDNA, AG, and 45S rDNA repeats. AAG showed strong hybridization signals on all of the seven chromosome pairs of $\mathrm{H}$. vulgare, while the signals appeared, with varying patterns, on only six pairs of $H$. chilense chromosomes (Figs. 1a and 1b). This suggests that different events of AAG-repeat amplification occurred in the evolution of $H$. chilense and $H$. vulgare. Likewise, hybridization signals for AG were observed only on the centromere of all $H$. vulgare chromosomes. However, no signal appeared on any chromosome of $H$. chilense (Figs. 1e and 1f).

Signals attributable to $5 \mathrm{~S}$ rDNA appeared on four pairs of chromosomes in $H$. vulgare, but on only one pair in $H$. chilense (Figs. $1 \mathrm{c}$ and $1 \mathrm{~d}$ ). On the other hand, the $45 \mathrm{~S}$ rDNA probe showed the same pattern on the two pairs of satellite chromosomes of both Hordeum species (Figs. 1e and 1f)

Allocation of $\boldsymbol{H}$. chilense chromosomes By comparison between the chromosomes in the original plant and those in the addition and substitution lines, the chromosomes of $H$. chilense, showing varied patterns of FISH signals, were allocated to the homoeologous groups (Figs. 2 and 3). AAG signals distinguished individual chromosomes $1 \mathrm{H}^{\text {ch }}$ to $7 \mathrm{H}^{\text {ch }}$ by their different patterns. No signals were observed on $6 \mathrm{H}^{\text {ch }}$, while the remaining six pairs showed signals of varying distribution and strength. Chromosome $3 \mathrm{H}^{\mathrm{ch}}$ showed the highest signal number and intensity, while $5 \mathrm{H}^{\mathrm{ch}}$ showed only one faint interstitial signal (Fig. 3). 5S rDNA signals appeared on the terminus of the short arm in the $H$. chilense chromosome of the $5 \mathrm{H}^{\text {ch }}$ addition line. 45S rDNA signals appeared on the short arm of addition lines $5 \mathrm{H}^{\text {ch }}$ and $6 \mathrm{H}^{\mathrm{ch}}$. The signal on $6 \mathrm{H}^{\text {ch }}$ was relatively closer to the centromere and stronger compared to that on $5 \mathrm{H}^{\text {ch }}$. No chromosome rearrangements were observed in the chromosomes in the addition or substitution lines.

Comparative FISH analysis of $\boldsymbol{H}$. chilense and $\boldsymbol{H}$. vulgare Pedersen et al. (1996) reported good agreement of the AAG-banding pattern of barley with the C-banding and N-banding patterns. In this study, individual chro- 

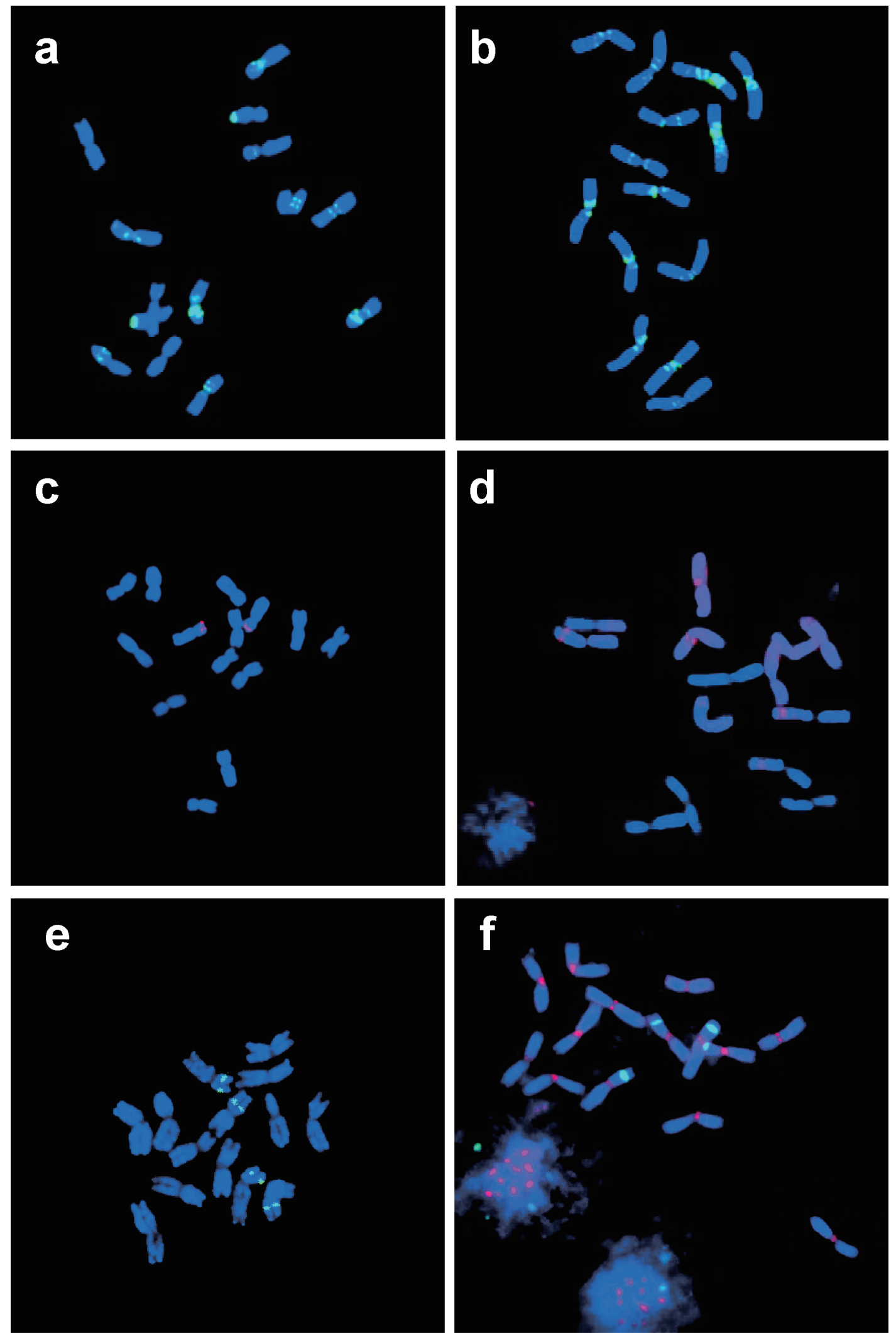

Fig. 1. FISH with AAG (a, b), 5S rDNA (c, d), and 45S rDNA (green) and AG (red) (e, f) to mitotic chromosomes of H. chilense (a, c, e) and $H$. vulgare $(\mathrm{b}, \mathrm{d}, \mathrm{f})$. 

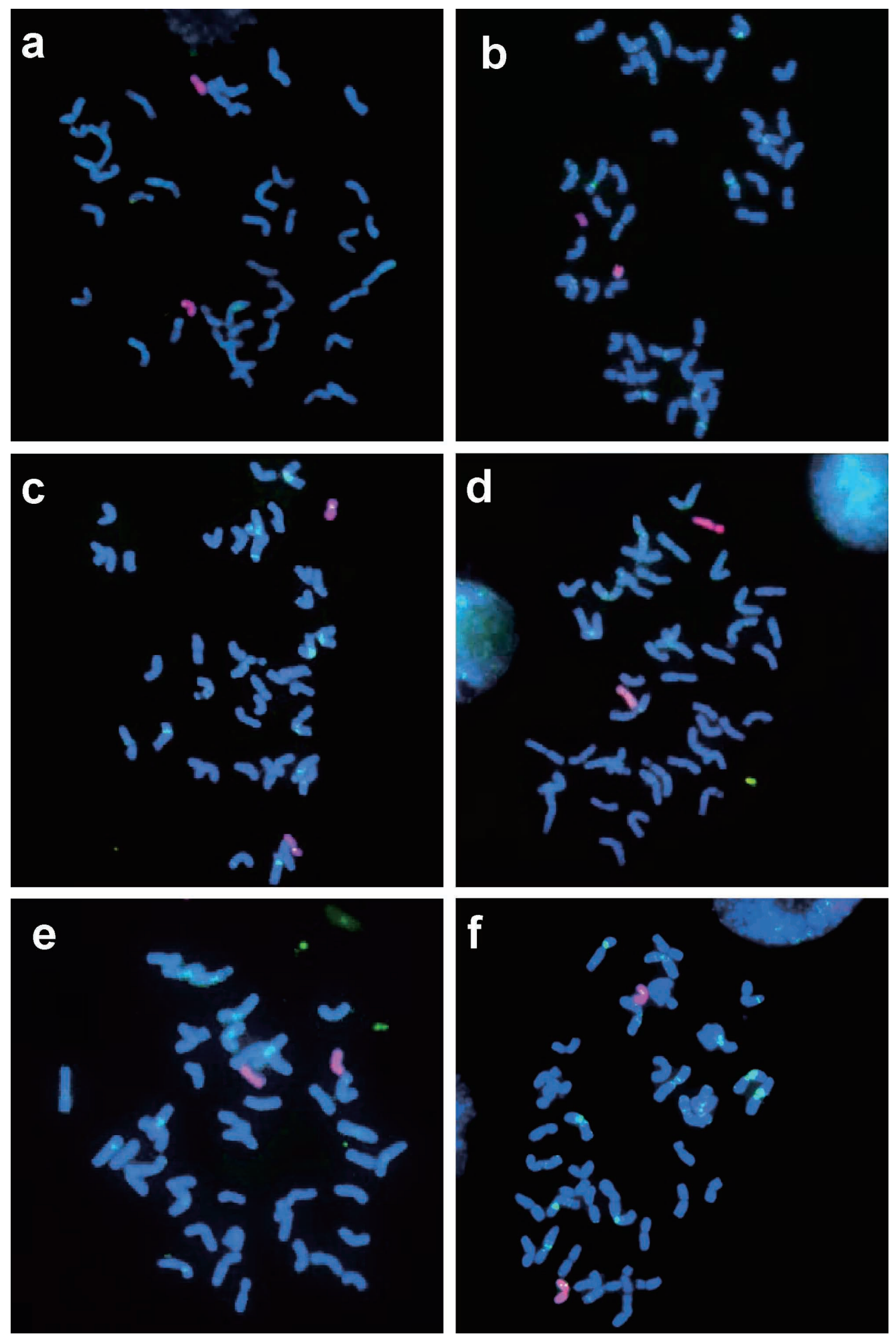

Fig. 2. Cytological characterization of $H$. chilense chromosomes with the AAG probe in wheat- $H$. chilense addition and substitution lines. (a) $1 \mathrm{H}^{\text {ch }}$ disomic substitution line. (b) $2 \mathrm{H}^{\text {ch }} \mathrm{S}$ telosomic addition line. (c) $4 \mathrm{H}^{\text {ch }}$ disomic substitution line. (d) $5 \mathrm{H}^{\text {ch }}$ disomic addition line. (d) $6 \mathrm{H}^{\mathrm{ch}}$ disomic addition line. (d) $7 \mathrm{H}^{\mathrm{ch}}$ disomic addition line. Wheat chromosomes appear blue and $H$. chilense chromosomes appear red. All lines except the $6 \mathrm{H}^{\text {ch }}$ disomic addition line showed AAG repeat signals (green). 


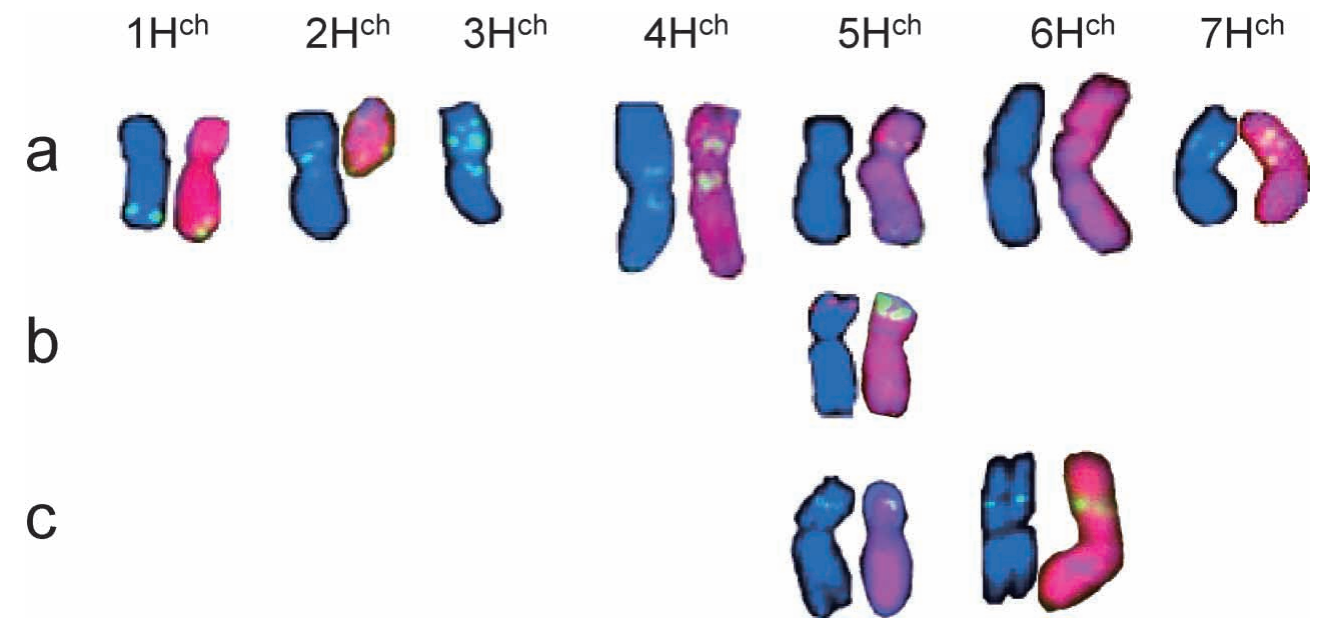

Fig. 3. Karyotyping of $H$. chilense chromosomes. The chromosomes of the original $H$. chilense are shown on the left side, and those of addition or substitution lines on the right side of each chromosome pair. (a) AAG repeat, (b) $5 \mathrm{~S}$ rDNA and (c) $45 \mathrm{~S}$ rDNA.

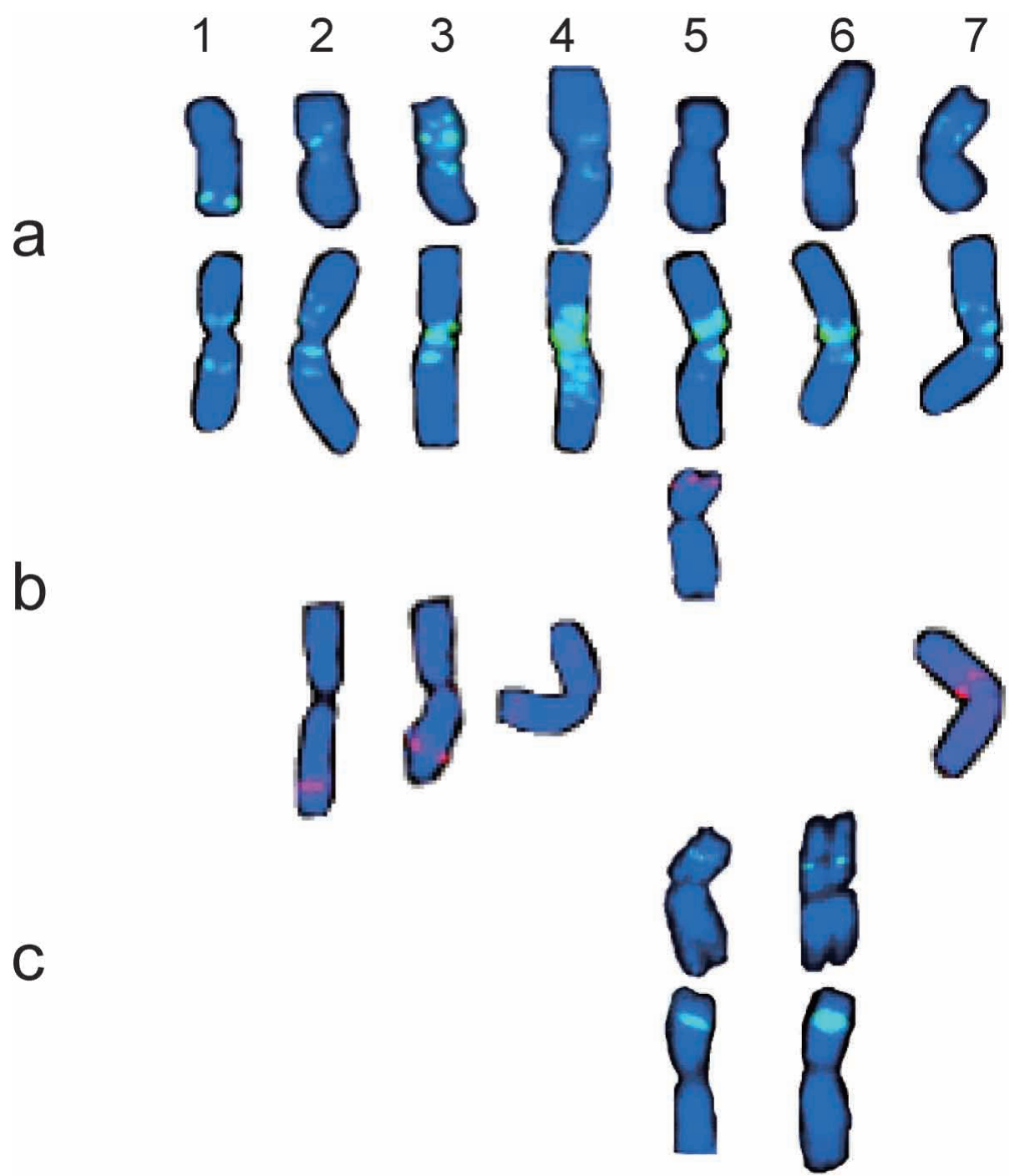

Fig. 4. FISH of $H$. vulgare and $H$. chilense with AAG (a), 5S rDNA (b) and 45S rDNA (c) repeats. For each chromosome pair the upper ones are of $H$. chilense and the lower ones are of $H$. vulgare. 
mosomes of barley were identified based on the ideogram of the C-banded chromosomes of barley cv. Betzes (Kakeda et al., 1991) and the AAG signal patterns obtained from this study. Likewise, in $H$. chilense AAG signals partly reflected the C-banding patterns presented by Cabrera et al. (1995). Fig. 4 shows a chromosomal comparison bet-

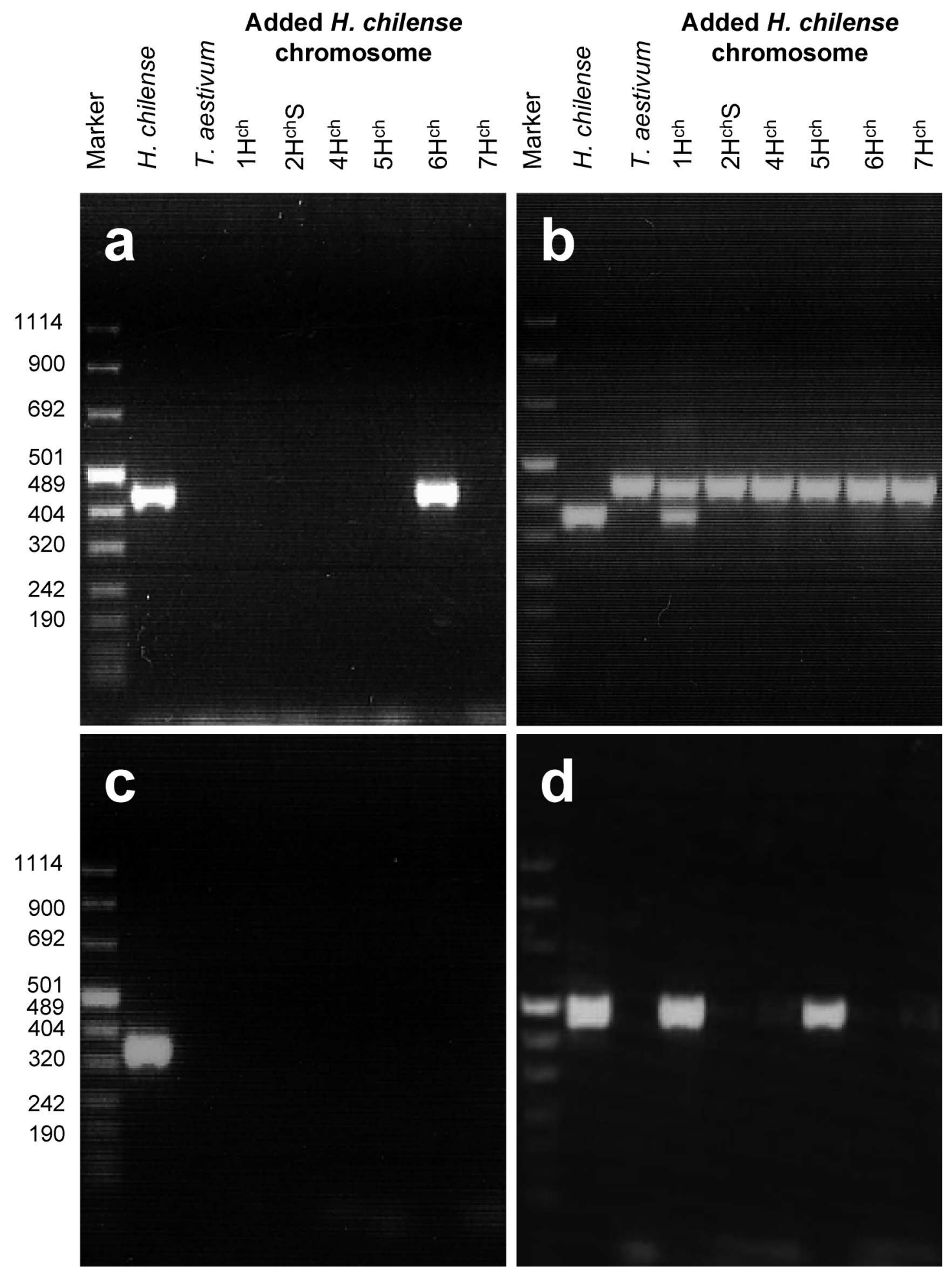

Fig. 5. Representative PCR amplification patterns. (a) Amplified fragments appeared only in $H$. chilense and one of the $H$. chilense addition/substitution lines (BAWU361). (b) Size-polymorphic bands appeared only in $H$. chilense and one of the $H$. chilense addition/ substitution lines (BAWU541). (c) A single band appeared only in H. chilense (BAWU414). (d) Amplified fragments appeared in $H$. chilense and two of the $H$. chilense addition/substitution lines (BAWU707). 
ween $H$. chilense and $H$. vulgare using FISH with three probes. The signal patterns of the AAG repeat differed between the homoeologous pairs of $H$. chilense and $H$. vulgare. None of the chromosomes of $H$. chilense showed a signal similar to that of the AAG repeats in $H$. vulgare. For instance, while chromosomes $1 \mathrm{H}$ and $4 \mathrm{H}$ of $\mathrm{H}$. vulgare showed two and many signals, respectively, the corresponding homoeologous pairs in $H$. chilense showed one and two signals (Fig. 4).

The locations of $5 \mathrm{~S}$ rDNA signals differed between the two species. They appeared on chromosomes $2 \mathrm{H}, 3 \mathrm{H}, 4 \mathrm{H}$ and $7 \mathrm{H}$ in $\mathrm{H}$. vulgare, but only on the nonhomoeologous chromosome $5 \mathrm{H}^{\mathrm{ch}}$ in $\mathrm{H}$. chilense (Fig. 4). As for $45 \mathrm{~S}$ rDNA, there was no difference in hybridization pattern between the two species, in both of which hybridization appeared on homoeologous chromosome groups 5 and 6 .

\section{Allocation of barley EST markers to H. chilense} chromosomes Among 209 markers generated in $H$. chilense, 140 markers were allocated to individual chromosomes (Table 2 and Fig. 5). Twenty-nine markers were on $1 \mathrm{H}^{\text {ch }}$ and $7 \mathrm{H}^{\text {ch }}, 26$ were on $4 \mathrm{H}^{\text {ch }}$ and $5 \mathrm{H}^{\text {ch }}, 21$ were on $6 \mathrm{H}^{\mathrm{ch}}$, and 10 on the short arm of $2 \mathrm{H}^{\mathrm{ch}}$. Seven markers were allocated to two or three chromosomes. For example, marker BAWU707 was allocated to chromosomes $1 \mathrm{H}^{\mathrm{ch}}$ and $5 \mathrm{H}^{\mathrm{ch}}$, and yielded an amplicon of the same size (Fig. 5d). Sixty-one primers amplified no PCR products with any of the used addition or substitution lines and hence these markers could not be allocated to specific chromosomes of $H$. chilense (Fig. 5c). This might be due to three reasons: the unavailability of addition lines of chromosomes $3 \mathrm{H}^{\text {ch }}$ and $2 \mathrm{H}^{\text {ch }} \mathrm{L}$, differences between accessions of $H$. chilense used in the production of the addition lines and the one used in this study, and differences in the most appropriate PCR conditions depending on whether the template DNA was from $H$. chilense or the wheat- $H$. chilense addition lines. Comparing the location of these markers on $H$. vulgare (Nasuda et al., 2005; Sato et al., unpublished data) and $H$. chilense chromosomes revealed a high degree of homology between the two species. All the markers allocated to chromosome $4 \mathrm{H}^{\text {ch }}$ of $H$. chilense were placed onto the homoeologous chromosome of $H$. vulgare. However, some of the markers were allocated to different homoeologous chromosomes. For example, two of the markers allocated to chromosome $5 \mathrm{H}^{\text {ch }}$ of $H$. chilense were placed on chromosome $2 \mathrm{H}$ and $3 \mathrm{H}$ of $\mathrm{H}$. vulgare, respectively.

\section{DISCUSSION}

Marked difference in chromosome morphology between $H$. chilense and $H$. vulgare Characterization of alien chromosomes is useful for their utilization in wheat improvement programs. Differentiation between the genomes of $H$. vulgare ssp. spontaneum and $H$. chil- ense was studied using repetitive sequences (Taketa et al., 2000). In that study, hybridization of the repetitive DNA sequence family pSc119.2 detected signals in $H$. chilense but not in $H$. vulgare ssp. spontaneum . Here, four probes of repetitive sequences for FISH and 209 barley EST markers were used to characterize the chromosomes of $H$. chilense and to compare their homoeology with the chromosomes of $H$. vulgare. Three out of the four probes (AAG, $5 \mathrm{~S}$ rDNA and $45 \mathrm{~S}$ rDNA) gave diverse signals that enabled us to distinguish individual chromosomes in both $H$. chilense and $H$. vulgare. The hybridization signals in $H$. chilense were conserved in all added or substituted $H$. chilense chromosomes in Chinese Spring wheat. This suggests that no detectable structural changes occurred in $H$. chilense chromosomes during the hybridization with wheat. On the other hand, the hybridization signals revealed differences at the chromosome level between $H$. chilense and $H$. vulgare. The centromeric DNA sequence differed between the two species: AG repeat signals were observed only in $H$. vulgare. In addition, AAG signals were observed on the chromosomes of the two Hordeum species but none of the homoeologous pairs showed the same signal pattern. Also, the hybridization signals of the $5 \mathrm{~S}$ rDNA repeat indicated differences in the numbers of chromosomes containing the repeat between the two species. Conservation of the number and position of signals between $H$. chilense and $H$. vulgare was observed only with the $45 \mathrm{~S}$ rDNA repeat. The usefulness of both $5 \mathrm{~S}$ and $45 \mathrm{~S}$ rDNA as phylogenetic markers in the genus Hordeum was previously suggested (de Bustos et al., 1996; Taketa et al., 1999). The physical locations of $5 \mathrm{~S}$ and $45 \mathrm{~S}$ rDNA in 15 diploid Hordeum species with 'I' genomes were examined (Taketa et al., 2000 and 2005). Those authors concluded that the rDNA sites are useful chromosome markers for investigations of the karyotype evolution and phylogeny of the I-genome species in the genus Hordeum.

Conservation of homoeology in $\boldsymbol{H}$. chilense and $\boldsymbol{H}$. vulgare In a previous study we proposed the usefulness of barley EST markers for the genetic analysis of ten alien Triticeae species related to wheat (Hagras et al., 2005). Out of 1,165 barley EST markers, 610 were transferable to $H$. chilense, and 292 of them were polymorphic between wheat and $H$. chilense. Of the 209 markers tested in this study, 140 were allocated to six pairs of $H$. chilense chromosomes using wheat- $H$. chilense addition and substitution lines. On average, 26 markers were allocated to each chromosome of $\mathrm{H}$. chilense. Most of these markers had already been allocated to $H$. vulgare chromosomes (Nasuda et al., 2005; Sato et al., unpublished data). In this study, $90 \%$ of the EST markers were allocated to the same homoeologous group of $H$. vulgare, indicating conservation of homoeology between the two species. The remaining markers were allocated to nonhomoeologous or 
multiple homoeologous groups. Non-conserved homoeology might be due to undetectable chromosome rearrangements that occurred during the evolution of the species. EST markers might be a good tool for detecting such chromosomal rearrangements.

EST markers have advantages over repetitive sequences in genome comparisons because they originate from expressed sequences and most of them can identify unique regions. Therefore, an EST is more likely to be conserved across species than repetitive sequences, and thus is more likely to be useful for comparative mapping. The degree of homology of the EST markers in H. chilense and $H$. vulgare indicates a close relationship between the two species, in spite of the great differences shown by FISH analysis using the four repetitive sequence probes. The EST markers were conserved while the repetitive sequences have rapidly changed during the evolution of the two species. Using ESTs is more reliable than using repetitive sequences to study the homology between these species.

\section{Use of allocated EST markers for genetic stud-}

ies The current knowledge about the genomes of wild Triticeae species is limited. Alien chromosome addition and substitution lines in wheat are important genetic resources for genetic studies on individual chromosomes of alien species related to wheat. Moreover, EST markers allocated to individual chromosomes or chromosome arms would make those genetic resources more useful. This study presented 140 EST markers specific to the $H$. chilense chromosomes. This large number of markers should encourage further efforts to construct a fine genetic map for this important resource. The repetitive sequences and EST markers allocated to the specific chromosomes of $H$. chilense in this study will be useful in identifying and tracing introgressed $H$. chilense chromosomes and chromosomal segments in a wheat background.

\section{REFERENCES}

Cabrera, A., Friebe, B., Jiang, J., and Gill, B. S. (1995) Characterization of Hordeum chilense chromosomes by C-banding and in situ hybridization using highly repeated DNA probes. Genome 38, 435-442.

De Bustos, A., Cuadrado, A., Soler, C., and Jouve, N. (1996) Physical mapping of repetitive DNA sequences and $5 \mathrm{~S}$ and 18S-26S rDNA in five wild species of the genus Hordeum. Chromosome Res. 9, 491-499.

Endo, T. R., and Gill, B. S. (1996) The deletion stocks of common wheat. J. Hered. 87, 295-307.

Friebe, B., Jiang, J., Raupp, W. J., McIntosh, R. A., and Gill, B. S. (1996) Characterization of wheat-alien translocations conferring resistance to disease and pest: current status. Euphytica 91, 59-87.

Friebe, B., Mukai, Y., Dhaliwal, H. S., Martin, T. J., and Gill, B. S. (1991) Identification of alien chromatin specifying resis- tance to streak mosaic and greenbug in wheat germplasm by C-banding and in situ hybridization. Theor. Appl. Genet. 81, 381-389.

Gale, M. D., and Miller, T. E. (1987) The introduction of alien genetic variation into wheat. In: Wheat breeding: its scientific basis (Lupton FGH ed). Chapman and Hall. pp. 173210.

Hagras, A. A., Kishii, M., Sato, K., Tanaka, H., and Tsujimoto, H. (2005) Extended application of barley EST markers for the analysis of alien chromosomes added to wheat genetic background. Breed. Sci. 55 (in press)

Hudakova, S., Michalek, W., Presting, G. G., ten Hoopen, R., dos Santos, K., Jasencakova, Z., and Schubert, I. (2001) Sequence organization of barley centromeres. Nucleic Acids Res. 29, 5029-5035.

Jiang, J., and Gill, B. S. (1994) New 18-26S ribosomal RNA gene loci: chromosomal land markers for the evolution of polyploid wheats. Chromosoma 103, 179-185.

Jiang, J., Friebe, B., and Gill, B. S. (1994) Recent advances in alien gene transfer in wheat. Euphytica 73, 199-212.

Kakeda, K., Fukui, K., and Yamagata, H. (1991) Heterochromatic differentiation in barley chromosomes reveled by $\mathrm{C}$ and N-banding techniques. Theor. Appl. Genet. 81, 144150.

Kishii, M., Nagaki, K., Tsujimoto, H., and Sasakuma, T. (1999) Exclusive localization of tandem repetitive sequences in subtelomeric heterochromatin regions of Leymus racemosus (Poaceae, Triticeae). Chromosome Res. 7, 519-529.

Knott, D. R., and Dvorak, J. (1976) Alien germplasm as a source of resistance to disease. Ann. Rev. Phytopathol. 14, 211235.

Leitch, I. J., Leitch, A. R., and Heslop-Harrison, J. S. (1991) Physical mapping of plant DNA sequences by simultaneous in situ hybridization of two differently labeled fluorescence probes. Genome 34, 329-333.

Martín, A. (1998) Tritordeum: the first ten years. Rachis 7: 1215

Martín, A., Cabrera, A., Hernandez, P., Ramirez, M. C., Gimenez, M. J., Rubiales, D., and Ballestros, J. (2000) Prospects for the use of $H$. chilense in durum wheat breeding. In: Broertjes C (ed) proc Int Symp Eucarpia on induced Variability in Plant Breeding PP. 79-81. pudoc, Wageningen.

Martín, A., Martín, L. M., Cabrera, A., Ramirez, M. C., Gimenez, M. J., Rubiales, D., Hernandez, P., and Ballestros, J. (1998) The potential of Hordeum chilense in breeding Triticeae species. In: Triticeae III. Jaradat, A.A. (ed.) Science publisher, Inc., Enfield, NH, USA, pp. 377-386.

Miller, T. E., Reader, S. M., and Chapman, V. (1982) The addition of Hordeum chilense chromosomes to wheat. In Induced Variability in Plant Breeding: Proceedings of an International Eucarpia Symposium, Wageningen, September 1981. Edited by C. Broertjes. Pudoc, Wageningen, The Netherlands, pp. 79-81.

Molnar-Lang, M., Lince, G., Friebe, B., and Sutka, J. (2000) Detection of wheat-barley translocations by genomic in situ hybridization in derivatives of hybrids multiplied in vitro. Euphytica 112, 117-123.

Mukai, Y., Endo, T. R., and Gill, B. S. (1990) Physical mapping of the $5 \mathrm{~S}$ rDNA multigene family in common wheat. J. Hered. 81, 290-295.

Mukai, Y., Friebe, B., and Gill, B. S. (1992) Comparison of Cbanding patterns and in situ hybridization sites using highly repetitive and total genomic rye DNA probes if Imperial rye chromosomes added to Chinese Spring wheat. J. Genet. 67, 71-83. 
Mukai, Y., Friebe, B., Hatchett, J. H., Yamamoto, M., and Gill, B. S. (1993) Molecular cytogenetic analysis of radiation induced wheat-rye terminal and intercalary chromosomal translocations and the detection of rye chromatin specifying resistance to Hessian fly. Chromosoma 102, 88-95.

Murray, H. G., and Thompson, W. F. (1980) Rapid isolation of high molecular weight plant DNA. Nucleic Acids Res. 8, 4321-4325.

Nasuda, S., Kikkawa, Y., Ashida, T., Rafiqul Islam, A. K. M., Sato, K., and Endo, T. R. (2005) Chromosomal allocation and deletion mapping of barley ESTs. Genes and Genetic Systems (in press)

Pedersen, C., Rasmussen, S. K., and Linde-Laursen, I. (1996) Genome and chromosome identification in cultivated barley and related species of the Triticeae (Poaceae) by in situ hybridization with the GAA-satellite sequence. Genome 39, 93-104.

Person-Dedryver, F., Jahier, J., and Miller, T. E. (1990) Assessing the resistance to cereal root-knot nematode, Meloidogyne naasi. I: a wheat line with the added chromosome arm $1 \mathrm{H}^{\text {ch }} \mathrm{S}$ of Hordeum chilense. J. Genet. Breed. 44, 291-296.

Sato, K., Nankaku, N., Yano, K., and Takeda, K. (2003) Large scale development of barley EST markers. Ikushugaku Kenkyu, 5 (suppl. 1): 93.
Sharma, H. C., and Gill, B. S. (1983) Current status of wide hybridization in wheat. Euphytica 32, 17-31.

Taketa, S., Ando, H., Takeda, K., Harrison, G. E., Heslop-Harrison, J. S. (2000) The distribution, organization and evolution of two abundant and widespread repetitive DNA sequences in the genus Hordeum. Theor. Appl. Genet. 100, 169-176.

Taketa, S., Ando, H., Takeda, K., Ichii, M., and Von Bothmer, R. (2005) Ancestry of American polyploid Hordeum species with the I genome inferred from 5S and 18S-25S rDNA. Ann. Bot. (published online on April 13, 2005).

Taketa, S., Harrison, G. E., Heslop-Harrison, J. S. (1999) Comparative physical mapping of the $5 \mathrm{~S}$ and $18 \mathrm{~S}-25 \mathrm{~S}$ rDNA in nine wild Hordeum species and cytotypes. Theor. Appl. Genet. 98, 1-9.

Tsujimoto, H., Yamada, T., and Abe, T. (2001) Chromosome breakage in wheat induced by heavy ion-beam irradiation. Riken Report 34, 172.

Tsujimoto, H., Mukai, Y., Akagawa, K., Nagaki, K., Fujigaki, J., Yamamoto, M., and Sasakuma, T. (1997) Identification of individual barley chromosomes based on repetitive sequences: Conservative distribution of Afa-family repetitive sequences on the chromosomes of barley and wheat. Genes and Genetic Systems 72, 303-309. 